\title{
Impact of religion and culture on mental disorders among Egyptians
}

\author{
Wafaa Gadelkarim, ${ }^{1}$ Manal El-Maraghy ${ }^{2}$ and Akeem Sule ${ }^{3}$
}

'Specialty Doctor, South Essex Partnership University NHS Foundation Trust, Essex, UK email wafaa.gadelkarim@sept.nhs.uk

${ }^{2}$ Consultant Psychiatrist, Derwent Centre, North Essex Partnership NHS Foundation Trust, Essex, UK ${ }^{3}$ Consultant Psychiatrist, South Essex Partnership University NHS Foundation Trust; Honorary Visiting Research Associate, Department of Psychiatry, Cambridge University, UK
This review looks at the long-established customs and religious beliefs, as well as social and family structures, affecting the symptoms of psychiatric disorders among Egyptian people. It considers reactions to emotional distress and dysfunctionality. Some light is also shed on the healthcare system.

Cross-cultural psychiatry is concerned with the cultural and ethnic expression of mental disorders. With increasing cultural diversity, an understanding of these issues has become essential to good clinical practice.

Egypt has a mixed Muslim and Christian population, and so its centuries of traditions are coloured with strong religious beliefs; this plays an important role in the psychological make-up of its people. Nonetheless, few studies have explored its impact on mental health. Abdel-Khalek (2011) concluded that a high score for religiosity is related to good mental health and feeling happier. Religion is able to bind members of society through their shared acceptance of a set of moral beliefs and values. Indeed, this can become a part of the everyday behaviour of non-believers as well as of believers (Okasha, 2010).

\section{Epidemiology}

Egypt - by virtue of its geographical location - is central to the Arab world, is the gateway to Africa and has strong links in the Mediterranean region. It has a population of over 80 million (15-20\% Christian), mostly living in the limited space along the bank of the Nile. The community structure still largely revolves around the extended family.

In spite of its wealth and natural and human resources, it is burdened by illiteracy and slow economic growth. Despite some improvement in standards of living, the average Egyptian remains poor. This, along with other factors there is not space to cover here, eventually led to the revolution on 25 January 2011.

An initial analysis of a recent national household survey produced an estimate for the prevalence of mental health disorder of $16.93 \%$, which is consistent with rates found worldwide (10-20\%). Mood disorders were the commonest $(6.43 \%)$, followed by anxiety disorders $(4.75 \%)$ and multiple disorders $(4.72 \%)$. The prevalence of psychosis was around $0.2 \%$, which is slightly lower than is commonly reported worldwide (Ghanem et al, 2009).

\section{Profile of psychiatric presentation}

Social and cultural factors affect symptom expression. Among Egyptian patients, depression is mainly manifested as agitation with somatic symptoms like poor libido, anorexia and insomnia. Patients tend to mask their affect with multiple somatic symptoms, probably because of the greater social acceptance of physical complaints.

Similarly, men with anxiety disorders mostly present with hypochondriacal complaints; in Egyptian culture 'real men' should not have psychological symptoms. Women, in contrast, tend to show free-floating anxiety and loss of weight (Okasha, 2004a).

Okasha (2004b) points out that the role of religious upbringing has been evident in the phenomenology of obsessive-compulsive disorder (OCD) in Egypt. The emphasis on religious rituals, in the form of repeated ablutions prior to prayer accompanied by the repetition of particular phrases, along with the warding off of blasphemous thoughts through repeated prayers could explain the high prevalence of religious obsessions and compulsions involving repetitions and cleanliness. The presentation of OCD among Egyptians is more similar to that in the Jewish population and somewhat different to that among Christian and Hindu populations. A surprising finding in Okasha's study was that insight was mildly affected in $26 \%$ of cases, moderately affected in $50 \%$ and severely affected in $14 \%$. This contrasted with the accepted characteristics of OCD that patients recognise the absurdity of their obsessions and compulsions.

In schizophrenia, religious delusions are frequent, in accordance with the highly religious nature of much of the Egyptian population. Often, beliefs include the intervention of supernatural beings, possession with jinn/spirits, occult forces or magic. Atallah et al (2001) studied the files of 913 Egyptian in-patients, and identified 632 sets of records that made reference to some religious or spiritual content in the patient's delusions. In total, 309 patients had religious delusions: $44 \%$ of these patients had delusions that involved God in some way, $24 \%$ believed they were victims of black magic and $22 \%$ believed that an evil spirit controlled them. Sexual delusions were also common, owing to the suppressive social and religious environment.

Presentation with catatonia is more frequent in Egypt than in other parts of the world, probably because of the delay in seeking treatment.

When it comes to substance misuse, a study at Ain Shams University by Khalil et al (2008) referred to some figures from the 2005 report of the United Nations Office on Drugs and Crime. It indicated that drug misuse in Egypt is most problematic among men aged $20-30$ years. It is less prevalent 
among women, although the incidence is on the rise; women are usually aged between 15 and 25 years when they do misuse substances. Women tend to come from higher social classes, and their preferred drugs are hypnotics and tranquillisers. The greater prevalence of substance misuse disorders among men is due to the greater freedom accorded to them.

Drug use is still regarded as a moral failure. The substances most commonly misused are cannabis preparations such as bango and hashish. Heroin misusers number around 20000-30000. Characteristically, in the sample under study, $64 \%$ were reported to be living with their families for most of the previous 3 years. Alcohol misuse was rare, not surprisingly, as alcohol use is prohibited by the Muslim faith. This is in contrast to the relative social acceptance of the use of cannabis, in the mistaken belief that it does not affect consciousness or judgement.

In relation to suicide, Okasha (2004a) stated that 'feelings of hopelessness and the intention to kill oneself were not common among Muslims, for whom losing hope in the relief by God and selfinflicted death are blasphemous and punishable in the afterlife'. That also applies to the conservative Coptic Christians. There is no formal statistical documentation of suicide in Egypt, as it is a taboo subject. Most cases of suicide and attempted suicide are due to depression and personality disorders. They are mostly through drug overdose, and these patients are generally referred to hospital toxicology departments, with no or scant recording of information such as whether the overdose was accidental or intentional. Worthy of note, one of the authors went back to Cairo to practise through a volunteer programme after 15 years abroad, and observed that younger patients spoke freely about suicidal ideas and self-harm behaviour.

Child and adolescent psychiatry has attracted more attention in Egypt of late. Despite the interest in attention-deficit hyperactivity disorder (ADHD), the recent National Survey of the Prevalence of Mental Disorder excluded symptoms of ADHD at its first stage (Ghanem et al, 2009). Pervasive developmental disorders are mostly seen by paediatricians rather than psychiatrists. An Egyptian study looking at risk factors for autism (El-Baz et al, 2011) found that autism is twice as common in boys as girls (with a sex ratio of up to 5:1 for higher-functioning autism). Interestingly, $48 \%$ of the study sample were of higher social class, with parents mostly professionals. Generally speaking, parents find it hard to accept that their offspring have difficulties and consider it a failure on their part when they eventually seek help.

\section{Mental health services in Egypt}

Treatment of mental illness in Egypt was documented on the Kahum papyrus, which dates to 1900 BC. Suggestion played an important role. One of the psychotherapeutic methods used was 'temple sleep', which involved the use of herbs, quite possibly opium (Okasha, 2001).
Nowadays, it is widely acknowledged that the stigma attached to mental illness often leads to its underestimation and delay in seeking help. In rural Egypt, it is culturally acceptable for the family rather than the individuals themselves to make the decision to seek help; in such circumstances it is less likely that help will be sought for a woman.

In a qualitative study, Coker (2006) collected data through in-depth interviewing of 209 lay people, 106 psychiatric patients and their families, and 26 psychiatric nurses and social workers, to determine local knowledge and beliefs regarding mental illness. The study revealed the complex nature of psychiatric stigma and that Egyptians rely on religious healers as well as psychiatrists.

Mental healthcare is mainly available within the public sector. Although there are private institutions whose patients are well supported by their family networks and a multidisciplinary approach, they are not affordable for the average Egyptian. For this group, second- and third-generation antipsychotics and antidepressants are available; and treatment results match international rates.

The mental health service in the public sector was seen by Jenkins et al (2010) as underresourced in terms of infrastructure and workforce. The total number of hospital beds for a population then of around 75 million was 6156 , including 788 forensic beds (i.e. one bed/12000 population), compared with recommendations of $5-8$ beds/ 10000 from the World Health Organization. In 2009, there were 979 registered psychiatrists, of whom 285 were consultants; however, these figures will not be accurate, because of emigration: Egypt has lost a high proportion of its psychiatrists to rich countries. Most of the specialists are concentrated in major centres.

In 2006, a public awareness project was started by the Mental Health Secretariat to address all of the above. One of the major achievements was the ratification of the Mental Health Act in May 2009 by the Egyptian Parliament, the first attempt since 1944. The new Act is now in force across all private and public centres, protecting patients' rights. The parliamentary debates and the accompanying media attention presented an opportunity to highlight the needs of psychiatric patients and the importance of care in the community (Loza, 2010).

\section{Conclusion}

There are similarities and differences in presentation as well as management and service provision in mental health in Egypt compared with Western countries. The new spirit in post-revolution Egypt may support an improvement in mental health services.

\section{References}

Abdel-Khalek, A. M. (2011) Associations between religiosity, mental health and subjective well-being among Arab samples from Egypt and Kuwait. Mental Health, Religion and Culture, 15, 741-758.

Atallah, S. F., El-Dosoky, A. R., Coker, E. M., et al (2001) A 22-year retrospective analysis of the changing frequency and pattern of religious symptoms among inpatients with psychotic illness in Egypt. Social Psychiatry and Psychiatric Epidemiology, 36, 407-415. 
Coker, E. (2006) Religion, morality and psychiatric stigma in psychiatry in Egypt. Arabpsychnet e-Journal, 10-11.

El-Baz, F., Ismael, N. A. \& Nour El-Din, S. M. (2011) Risk factors for autism: an Egyptian study. Egyptian Journal of Medical Human Genetics, 12, 31-38

Ghanem, M., Gadallah, M., Meky, F. A., et al (2009) National Survey of Prevalence of Mental Disorders in Egypt: preliminary survey. Eastern Mediterranean Health Journal, 15, 65.

Jenkins, R., Heshmat, A., Loza, N., et al (2010) Mental health policy and development in Egypt - integrating mental health into health sector reforms 2001-9. International Journal of Mental Health Systems, 4, 17

Khalil, A., Okasha, T., Shawky, M., et al (2008) Characterization of substance abuse patients presenting for treatment at a university psychiatric hospital in Cairo, Egypt. Addictive Disorders and Their Treatment, 7, 199-209.

Loza, N. (2010) Integrating Egyptian mental health services into primary care: the policy maker's perspective. International Psychiatry, 7, 5

Okasha, A. (2001) Egyptian contribution to the concept of mental health. Eastern Mediterranean Health Journal, 7, 1-4.

Okasha, A. (2004a) Focus on psychiatry. British Journal of Psychiatry, 185, 266-272.

Okasha, A. (2004b) OCD in Egyptian adolescents: the effect of culture and religion. Psychiatric Times, 21(5), 21-24.

Okasha, A. (2010) Religion and mental health in Islam. In Religion and Psychiatry: Beyond Boundaries (eds P. J. Verhagen, H. M. Van Praag, J. J. López-Ibor Jr, et al), pp. 1-45. Wiley.

\section{The Royal College of Psychiatrists' International Congress

$$
\text { 2 - } 5 \text { July } 2013
$$ \\ Edinburgh International Conference Centre, UK}

\section{Psychiatry in Ascent}

This year's Congress will provide overviews, workshops and training on important areas in the science, policy and practice of psychiatry. A full programme is available to view online. Highlights include a whole day of traineefocused lectures plus keynote lectures from:
- Professor Andreas Myer-Lindberg (Heidelberg)

- Dame Fiona Caldicott (Oxford)

- Professor Eve Johnstone (Edinburgh)

- Baroness Sheila Hollins (London)

- Professor Sir David Goldberg (London)

- Professor Sir Simon Wessely (London)

New from RCPsych Publications
By Valsamma Eapen, Philip Graham and Shoba Srinath
Mental health problems and worries are common among infants, children and adolescents in every part of the world. This
in places is a practical manual for primary healthcare professionals, teachers and anyone who works with children - especially
After giving the reader an overview of child mental health problems, the manual goes on to deal with the various developmental,
behavioural and emotional problems that arise in as many as 10\% of the youth population. For each problem it first
provides a case study and then describes how to find out more about a child with this problem.
It suggests what can be done to help the child and their family.
It also examines the mental health aspects of situations such as when children are maltreated
or exposed to natural or man-made disasters.
- Covers problems that health workers and teachers in low- and middle-income countries
often have to deal with.
- Written in simple language with many case examples and pictures.
The only comprehensive book on mental healthcare in young people for those with no
access to specialist medical advice.
Oct 2012, paperback, 214 pages, ISBN 978-1-908020-48-2, price £10
(RCPsych members' price: £8)

